\title{
La pédagogie de l'environnement pour lutter contre les conduites à risques
}

David Kumurdjian

\section{(2) OpenEdition \\ Journals}

Édition électronique

URL : http://journals.openedition.org/ere/6586

DOI : 10.4000/ere.6586

ISSN : 2561-2271

Éditeur

Centr'ERE

Référence électronique

David Kumurdjian, «La pédagogie de l'environnement pour lutter contre les conduites à risques », Éducation relative à l'environnement [En ligne], Volume 3 | 2002, mis en ligne le 20 mars 2002, consulté le 16 avril 2021. URL : http://journals.openedition.org/ere/6586; DOI : https://doi.org/10.4000/ere. 6586

Ce document a été généré automatiquement le 16 avril 2021 


\title{
La pédagogie de l'environnement pour lutter contre les conduites à risques
}

\author{
David Kumurdjian
}

1 Le Centre d'Expérimentation Pédagogique de Florac, établissement public national de l'enseignement agricole, est spécialisé dans la formation continue des personnels sur le paysage, l'écologie, la communication, la pédagogie, le territoire et la ruralité, la pratique d'activités de pleine nature. Sans être situé en banlieue urbaine ni au cœur des violences sociales, n'ayant aucune connaissance sur les drogues ou sur la prévention des conduites à risques auprès des adolescents, l'équipe a néanmoins participé à une opération proposée par la Mission Interministérielle de Lutte contre la Drogue et la Toxicomanie (MILDT). La réflexion pédagogique a pris pour cadre une opération pluriannuelle lancée par la MILTD, elle fut l'occasion pour l'équipe de mener une recherche participative avec des équipes d'enseignants motivés sur l'utilisation de pédagogies de l'environnement au service de la socialisation des groupes d'apprenants en vue de projets d'éducation à la santé.

2 L'idée de départ était d'accompagner des équipes éducatives de lycée agricole dans un projet d'éducation à la santé qui passe par la constitution d'un groupe-classe, d'une relation éducative se construisant sur la confiance, la communication entre les élèves et les éducateurs.

3 Il s'agit donc, avant de proposer des apprentissages sur les savoirs (une instruction), de (ré)installer une vie sociale dans la classe qui ait un sens pour les acteurs (socialisation qui participe alors d'une éducation).

4 Nous avons formulé l'hypothèse que l'environnement, notamment son approche sensible, peut constituer un support de formation pour initier des relations éducatives positives entre les membres du groupe qui vivra une expérience d'éducation relative à l'environnement (ERE). Il s'agissait donc d'utiliser le champ pédagogique propre à l'éducation relative à l'environnement au service d'un projet à caractère social. 


\section{Pourquoi et comment concilier des objectifs d'éducation relative à l'environnement et des objectifs d'éducation à la santé ?}

Nous décrirons ici :

- deux tendances pédagogiques de l'ERE (éducation à l'environnement et éducation par l'environnement ;

- les priorités d'un partenaire nouveau (la MILDT) et la nouvelle problématique qu'il formule afin d'atteindre ses objectifs (travail de socialisation ;

- à partir de ces fondements, l'hypothèse que l'éducation par l'environnement peut participer aux objectifs de socialisation et de prévention des risques que s'est donnée la mission interministérielle.

\section{Une pédagogie qui utilise l'environnement comme support de formation}

6 L'éducation relative à l'environnement peut se comprendre et se pratiquer selon deux grands objectifs: éducation à l'environnement (l'environnement est objet d'étude), éducation par l'environnement (l'environnement est support de formation). Lorsque le projet éducatif suit l'axe de l'éducation par l'environnement, les approches pédagogiques esthétiques et physiques, regroupées généralement sous le terme d'approche sensible, en constituent le noyau central. Dans ce cas-là, la posture des éducateurs, l'énergie, les outils et les démarches pédagogiques serviront des objectifs centrés sur l'apprenant, sur le sujet, sur le groupe. On visera avant tout l'expression des individus, la socialisation, le plaisir de vivre et, ou d'apprendre.

7 L'utilisation d'activités d'expression, d'activités de pleine nature (APN) et d'une pédagogie de projet peuvent répondre parfaitement à cet objectif. Il s'agit de transformer les émotions et les énergies éprouvées par les adolescents en difficulté au moyen du contact avec les éléments naturels (l'eau, la roche, la falaise, le vent, la grotte) en éléments de plaisir et de connaissance, sources de progrès pour le groupe.

8 On chemine ainsi du plaisir des personnes et des groupes à la satisfaction de communiquer dans un climat de confiance et de bien-être. On passe du ressenti des émotions et de l'activité corporelle au plaisir de l'expression et de la communication à l'aide d'ateliers centrés sur la créativité artistique.

9 Dominique Cottereau (1994), à propos du séjour d'éducation à l'environnement, résume les effets induits d'une pédagogie de l'environnement qui se déroule hors de la classe, pendant une classe de découverte ou un séjour :

Les désirs, les énergies, les engagements de chaque enfant se focalisent sur la vie collective et le tissu relationnel qu'elle réorganise. De nouveaux aspects de personnalité se laissent découvrir, des liens se renforcent, se créent ou disparaissent [...]. Une promenade sur le chemin côtier n'a rien d'un moment de silence attentif à la nature, au contraire les langues vont bon train : on se raconte, on se souvient, on projette, on conciliabule, on parle de tout sauf de l'environnement. 


$$
\begin{aligned}
& \text { éducative qui génère, autant qu'elle s'appuie dessus, la confiance entre les participants } \\
& \text { (jeunes ou adultes). }
\end{aligned}
$$

\section{Les conduites à risques et la MILDT}

La Mission Interministérielle de Lutte contre les Drogues et les Toxicomanies (MILDT) a défini un plan triennal (1999/2001) autour de plusieurs objectifs (MILDT, 1999).

12 La plupart des objectifs sont centrés sur la connaissance des produits et leurs consommations : études, soins, formation des professionnels, répression du trafic, etc. Le propre de cette façon de réguler ou résoudre les problèmes de drogues et de toxicomanie, que ce soit pour gérer la consommation ou la répression du trafic, c'est de s'intéresser au produit et non aux individus qui le consomment.

3 Une autre des orientations données par la MILDT, qui elle est nouvelle, sous l'impulsion du professeur Parquet (1999), a défini des objectifs centrés sur le sujet, qui viennent compléter le plan d'action. Ils visent notamment à mieux comprendre les comportements et développer une socialisation entre jeunes et adultes, notamment au profit des jeunes scolarisés.

Plusieurs pistes ont ainsi été proposées dans ce plan triennal (MILDT, 1999, p. 36) :

- Le rappel de l'interdit (rapport à la loi et au droit). La conduite à risque n'est pas interdite par la loi, mais les consommations sont réglementées et il y a des différences entre, par exemple, l'alcool, le tabac et l'ecstasy.

- Une information sur les produits, mais aussi sur les dommages, c'est-à-dire les conséquences sociales et sanitaires qui font que le risque est réel et qu'il peut porter préjudice au sujet, mais également par contre coup à la société : responsabilité collective, marginalisation, etc.

- Un travail visant à développer les facteurs de protection (estime de soi, compétences personnelles, etc.) et à prendre en compte les facteurs de vulnérabilité propres à des catégories de la population ou à des individus.

15 Ainsi, à travers ces propositions, la MILDT invite des éducateurs, des enseignants, des professionnels travaillant en milieu éducatif (surveillants, infirmières scolaires, conseillers d'éducation, etc.) à s'intéresser au problème des conduites à risques qu'ils peuvent rencontrer dans leurs établissements avec les élèves, et propose une entrée éducative pour participer à la lutte qui ne nécessite pas de compétences fortes sur les produits. Car :

l'école est très impliquée. Ce n'est pas elle qui crée la violence, ni la consommation d'alcool, de tabac ou de drogue, mais la manière dont l'élève va vivre sa scolarité, s'il est en situation de réussite ou d'échec, s'il a le sentiment que son orientation est satisfaisante ou non, les relations qu'il y entretiendra avec les adultes, tout ceci sera fortement lié à son engagement dans des conduites délictueuses ou de consommation. (Ballion, 1999)

Éducation relative à l'environnement, Volume 3 | 2002 


\section{Vers une convergence théorique}

En définitive, il apparaît sur le plan théorique qu'une convergence d'objectifs est possible entre ces deux projets :

- d'une part, par l'émergence de nouvelles orientations sur la prévention des risques centrées sur la personne, le comportement individuel et en groupe entre jeunes, et entre jeunes et adultes, notamment dans l'institution scolaire;

- d'autre part, parce qu'en faisant le choix d'une éducation par l'environnement, où l'environnement est support de formation, la pédagogie est centrée sur la personne et non sur l'objet d'étude.

17 L'hypothèse est alors posée : une pédagogie de l'environnement qui repose sur des activités de pleine nature et d'expression peut participer à un apprentissage du vivre ensemble dans l'école, au sein de la classe et à la construction d'une image de soi positive. Cette socialisation positive peut permettre la réduction des conduites à risques (consommation de drogues, comportements agressifs).

\section{Le Projet d'Éducation Pour la Santé (PEPS)}

18 À partir de cette hypothèse, l'équipe du centre d'expérimentation décidait d'utiliser les deux constats pédagogiques suivants pour initier des projets d'éducation à la santé dans des lycées agricoles :

- les activités de pleine nature (APN) et la pédagogie de projet ont un effet socialisant sur le groupe classe, sur chaque élève, et améliorent les relations entre adultes (éducateurs, enseignants) et apprenants (élèves) ;

- des animateurs, des formateurs professionnels d'une pédagogie de l'environnement qui s'appuie sur des APN et des activités d'expression peuvent servir des projets d'enseignants autour de l'éducation à la santé.

19 Afin d'opérationnaliser le projet, le stage activités de pleine nature et expression, point de départ d'un Projet d'Éducation Pour la Santé (PEPS) discuté et construit avec une équipe dans un établissement, devrait participer à l'apprentissage du vivre ensemble dans l'école et au sein de la classe, et à la construction d'une image de soi positive pour chaque élève.

Le projet mené avec six équipes de lycées agricoles sera présenté en quatre parties : les différentes phases de l'action, la situation de départ, les deux objectifs noyaux visés et la méthodologie du stage.

\section{Les différentes phases de l'action}

Quatre étapes se sont succédées pour réaliser l'action :

- Appel d'offre lancé par la MILDT et relayé au CEP (Centre d'Expérimentation Pédagogique) par son administration de tutelle, la DGER (Direction Générale de l'Enseignement et de la Recherche, Ministère de l'Agriculture) au printemps 1999.

- L'équipe du CEP construit un dossier et élabore une méthodologie en concertation avec des équipes d'établissements intéressées. Deux sources sont utilisées pour contacter les équipes : notre réseau d'enseignants venus en formation continue sur des stages d'APN ou approche 
sensible du paysage, notre réseau d'enseignants ou de Conseiller Principaux d'Éducation venus en formation continue sur l'éducation à la citoyenneté ${ }^{1}$.

- Six équipes d'enseignants (pour autant de classes) sont ainsi contactées individuellement. Les six équipes préparent leurs projets. Comme point de départ de remédiation, elles acceptent notre offre, et la proposent à leurs classes. Le stage doit donner une cohésion à la classe, permettre de définir les règles, les devoirs et les droits des élèves, de mettre en relation le plaisir et l'effort, faciliter la confiance de l'élève en l'enseignant. Les stages avec les élèves ont lieu au CEP (quatre durant l'automne 99, deux au printemps 2000).

- Le CEP organise un regroupement de toutes les équipes (printemps 2000) pour faire le bilan et l'analyse des opérations PEPS dans chaque établissement.

\section{Le constat des équipes}

Afin de préparer des projets d'éducation à la santé, nous avons consulté les équipes sur «l'état des lieux» de leur classe (voir aussi le tableau 1). Avant de venir à Florac, les enseignants estiment que les élèves ont des conduites qui gênent le bon fonctionnement de l'école; les enseignants les voient passifs, souvent absents, agressifs ce qui leur fait supposer qu'ils consomment des substances interdites.

Tableau 1 : Les principales revendications ou observations des adultes avant le stage

\begin{tabular}{|c|c|}
\hline Les élèves manifestent : & Les équipes veulent: \\
\hline $\begin{array}{l}\text { 1. un évitement de la contrainte, } \\
\text { 2. une saturation, ils n'ont envie de rien, } \\
\text { 3. une surconsommation généralisée qui se } \\
\text { traduit principalement par une boulimie ou } \\
\text { par un suivi des modes, } \\
\text { 4. une non-socialisation des élèves qui se } \\
\text { cachent derrière des refuges identitaires, } \\
\text { des clans et des groupes, } \\
\text { 5. de nombreux non-dits, } \\
\text { 6. une déviance par rapport à la norme et des } \\
\text { conduites qui portent atteinte à leur santé. }\end{array}$ & $\begin{array}{l}\text { 1. que les élèves deviennent motivés, } \\
\text { 2. que les élèves retrouvent confiance en eux, } \\
\text { 3. lutter contre le tabagisme et les toxicomanies, } \\
\text { 4. lutter contre les drogues, contre la violence, } \\
\text { 5. mener une réflexion sur les conduites à } \\
\text { risques. }\end{array}$ \\
\hline
\end{tabular}

Les enseignants mettent cela sur le compte de l'ennui. Ils pensent que les élèves se font de mauvaises représentations de l'école dues au fonctionnement pédagogique de celleci sans pédagogie de projet, sans prise de risque dans la relation adulte/adolescent, sans communication, en incitant les élèves principalement à consommer l'école, non à participer.

\section{Les deux objectifs noyaux}

Chaque équipe d'établissement avait son propre projet. Deux points communs entre l'ensemble des équipes ont alors permis à l'équipe du CEP travailler sur l'hypothèse pédagogique que nous avions posée :

- Venir suivre un stage d'une semaine avec une classe d'élève à Florac et utiliser ce «moment » pour articuler le PEPS. Le stage vise à développer chez ces élèves, à partir d'activités de pleine nature très variées (spéléologie, canoë, escalade, randonnée à pied ou à vélo, etc.), leurs capacités d'expression et de communication : ateliers d'écriture, de poésie, 
d'expression corporelle, de théâtre, mime, graphisme, peinture, musique et sons, etc. Les règles de vie sont discutées avec les élèves en début de semaine. Il est mené en pédagogie de projet par l'équipe du CEP et coanimé par les enseignants.

- Participer au regroupement des équipes. Ce regroupement avait trois objectifs : 1) analyser et échanger sur les effets du stage de Florac dans le lycée d'un point de vue social et éducatif ; 2) analyser et échanger les expériences menées par chaque équipe autour de l'éducation à la santé ; 3) définir l'usage des drogues chez les jeunes et les conduites à risques (interventions d'un socioanthropologue et du professeur Parquet, pédopsychiatre).

C'est également à l'occasion du regroupement que nous avons pu entendre et recueillir les expériences de chaque équipe et en tirer des éléments d'évaluation.

\section{Méthodologie utilisée pour mener le stage APN-expression}

La méthodologie utilisée pour mener le stage à Florac est la pédagogie de projet. Habituellement centrée sur un objet d'étude, la pédagogie de projet est ici le support d'une éducation centrée sur l'élève. La pédagogie de projet comme outil d'ERE peut se décrire en quatre phases ${ }^{2}$ : contact, rebond, projet et restitution.

La phase contact consiste en une découverte du milieu par divers moyens d'approches sensibles et rationnels (activités de plein air, dessins, écriture, etc.) et permet d'initier des projets.

Le rebond, étape essentielle du choix de nouveaux projets par les élèves. Par groupes, et avec l'aide des enseignants et des animateurs du CEP, les élèves réalisent ainsi leur projet. Ce projet s'appuiera sur une activité physique de pleine nature. Le contrat pédagogique passé entre l'animateur et les élèves stipule que chaque groupe doit communiquer aux autres son expérience.

29 La préparation de la restitution et la restitution permettent à chaque groupe de finaliser et de communiquer une partie de cette expérience avec divers moyens d'expression (entre autres: vidéo, montage diapo, photo noir et blanc, CD-ROM, affiches, expression corporelle, théâtre, journal, etc.). Cette restitution est un facteur de motivation. C'est le moyen direct de valoriser le sujet dans son projet.

\section{Résultats}

Au total, les six équipes accompagnées des six classes sont venues suivre un stage d'une semaine et ont saisi cette occasion pour initier des PEPS.

Deux bilans qui font état des résultats sont présentés :

- Celui des équipes éducatives. Il permet de sentir la richesse et la diversité des actions et des réflexions, les remises en cause et les questions soulevées.

- Celui des élèves et qui traite du stage "plein air/expression ». Celui-ci est une synthèse de chaque bilan effectué pour chaque classe.

\section{Le bilan des équipes éducatives}

Ce bilan a été réalisé lors du regroupement à partir d'entretiens enregistrés avec chaque équipe à partir de deux questions : «Quel effet a eu le stage sur la classe et l'équipe ? » et « Quel effet a eu le stage dans l'établissement?». 


\section{Les effets sur la classe et l'équipe}

Nous en présentons une synthèse organisée autour de cinq items dans le tableau 2.

Tableau 2 : Les effets sur la classe et l'équipe

\begin{tabular}{|c|c|}
\hline Les élèves deviennent acteurs & Les relations entre les élèves se sont \\
\hline $\begin{array}{l}\text { Ils ont réalisé des dialogues et des } \\
\text { images, ils ont décrit et écrit ce qu'ils } \\
\text { avaient vécu, confectionné des panneaux, } \\
\text { un CD-Rom photos. Ils ont préparé des } \\
\text { restitutions pour les familles, se sont } \\
\text { « mis en scène ". La parole, } \\
\text { habituellement refoulée, a trouvé son } \\
\text { espace à travers des réalisations } \\
\text { concrètes : elle est valorisée. }\end{array}$ & $\begin{array}{l}\text { On a l'impression d'une prise de } \\
\text { connaissance mutuelle, de la création } \\
\text { d'une vie commune. Les filles ont réagi } \\
\text { immédiatement, les garçons ont réagi } \\
\text { plus lentement. Les élèves } \\
\text { communiquent, règlent leurs problèmes } \\
\text { rapidement, il y a une meilleure écoute } \\
\text { entre les élèves et moins d'agression } \\
\text { verbale. Des groupes se structurent. }\end{array}$ \\
\hline Les élèves sont responsabilisés & $\begin{array}{c}\text { Les relations des élèves avec les enseignants } \\
\text { ont changé }\end{array}$ \\
\hline $\begin{array}{l}\text { Les enseignants estiment que les élèves } \\
\text { ont développé leur autonomie, une } \\
\text { liberté de s'organiser, une prise } \\
\text { d'indépendance et de responsabilités. }\end{array}$ & $\begin{array}{l}\text { La relation élèves/professeurs s'est } \\
\text { humanisée, les élèves font davantage } \\
\text { confiance aux enseignants, qui ont } \\
\text { rapidement identifié chaque } \\
\text { élève/individu. On assiste, à partir d'un } \\
\text { vécu commun, à l'émergence d'une } \\
\text { culture commune. }\end{array}$ \\
\hline \multicolumn{2}{|c|}{$\begin{array}{c}\text { Les enseignants qui ont participé découvrent de nouvelles pratiques et innovent } \\
\text { pédagogiquement }\end{array}$} \\
\hline \multicolumn{2}{|c|}{$\begin{array}{l}\text { Ils ont mis en place des outils communs, un cahier de liaison, une concertation, une } \\
\text { gestion du temps pour se rencontrer. Certains expérimentent une graduation dans les } \\
\text { sanctions, une concertation avant de sévir, un cahier de projets professionnels, la double } \\
\text { correction (deux enseignants pour une copie) afin d'améliorer la situation de réussite } \\
\text { pour l'élève. }\end{array}$} \\
\hline
\end{tabular}

\section{Dans l'établissement}

Deux autres indicateurs qualitatif (la diversité des acteurs et partenaires) et quantitatif (le nombre de personnes touchées par l'action PEPS) de résultats ont permis d'évaluer l'action.

En effet, au départ, il s'agit d'un noyau restreint (2 à 5 enseignants) qui va ensuite en s'étoffant:

- à Hyères, onze enseignants se mobilisent dont le Conseiller Principal d'Education ;

- à Vervins, cinq personnes dont le chauffeur sont épaulés par un journaliste, un adjudant de gendarmerie, une infirmière spécialisée, un professeur d'arts martiaux ;

- à Saint-Gervais d'Auvergne, deux professeurs seuls au départ sont rejoints par l'infirmière, le CPE et une troupe de théâtre extérieure ;

-à Nérac, les quatre enseignants ont été appuyés par l'infirmière, une tabacologue, le comité de prévention contre l'alcoolisme ;

- à Montauban, au départ, deux enseignants et un auxiliaire (ATOSS) sont épaulés par le Proviseur adjoint, une enseignante en Éducation socioculturelle, l'infirmière et le chauffeur.

Ces personnes, largement motivées et impliquées dans le projet, ont contribué à forger des communautés éducatives accessibles aux discours de la prévention des conduites à risques et à la prise en compte de la santé par l'école. 


\section{Le bilan des élèves}

37 Nous n'avons recueilli que les bilans des élèves (environ 150 adolescents) sur le stage APN et expression qui s'est déroulé à Florac. Il n'y a pas eu de bilan élève sur l'opération PEPS globale menée dans les lycées.

Chaque grille de bilan de stage a été construite par l'équipe de l'établissement présente au stage. Aussi, les élèves de chaque classe ont-ils dû remplir des grilles différentes. Pour en faire la synthèse, nous avons organisé les réponses en utilisant cinq entrées. Deux entrées plutôt ouvertes : la réponse à la question "qu'est-ce que j'ai aimé? " doublée d'une liste de mots-clés à choisir. Trois entrées pour évaluer des objectifs : sur le stage et ses répercussions sur la scolarité, sur la discipline et les règles de vie, sur la consommation de tabac.

\section{Ce qu'ils ont aimé : les mots clés récurrents}

Trois types de réponses se dégagent pour les élèves :

- un autre cadre relationnel: vie de groupe bien vécue, une meilleure relation entre professeurs et élèves. Ainsi, ils écrivent : j'ai rencontré ma classe et expriment leur plaisir d'avoir rencontré les professeurs ;

- ils reconnaissent que ce sont essentiellement les activités d'expression et d'APN qui permettent la rencontre, l'échange, etc. quand elles sont pratiquées dans le plaisir ;

- ils expriment leur intérêt pour apprendre (sur les éléments, l'écologie, le paysage, etc.) en relevant qu'un tissu relationnel s'installe à partir de la réalisation de leur projet.

Les mots clés : deux familles de mots sont choisis par les élèves. Les mots centrés sur les éléments et les sensations associées : roche, froid, chaud, grotte, eau, vent, liberté, sécurité, aventure, plaisir, etc. Les mots centrés sur des valeurs : solidarité, bonheur, etc.

\section{Questions visant à vérifier des objectifs}

Sur le stage et ses répercussions sur la scolarité :

- On a formé un groupe, voici la phrase récurrente. Le vécu du stage et la constitution du groupe-classe apparaissent comme un préalable au travail «scolaire»: ils ont permis une meilleure connaissance des autres pour mieux travailler (élèves-élèves), et une plus grande confiance envers les adultes qu'ils souhaitent cultiver toute l'année.

- Cette idée forte doit pourtant être nuancée car la meilleure connaissance des individus a deux conséquences : des amitiés naissent, mais également des distances.

- Enfin, sur l'acte d'apprendre et la motivation, parmi toutes les phrases, citons celle-ci : Maintenant, on va s'amuser à faire un exposé !

Sur la discipline et les règles de vie de la semaine, les élèves ont apprécié le fait de pouvoir négocier. Ils ont été marqués et agréablement surpris qu'on leur donne la possibilité de discuter la Loi.

La consommation de cigarettes : cette question n'a été posée qu'à deux classes. Le nombre restreint de réponses nous empêche donc de formuler des conclusions. On notera cependant que la consommation a baissé pendant le stage, les élèves affirmant que c'est parce qu'ils étaient plus occupés, ou qu'ils n'avaient pas le temps de s'ennuyer. 


\section{Conclusion}

Les enseignants et éducateurs qui ont participé à l'opération PEPS n'ont pas de "gros » problèmes avec leurs élèves. Les symptômes sont la passivité, l'absentéisme, l'intolérance, une certaine agressivité, la probable consommation de substances interdites. Mais ils sont toutefois conscients de la responsabilité de l'école (l'ennui, l'absence de pédagogie active, de gestion du risque, difficultés de communication), conscients que ces éléments peuvent participer au malaise des élèves et développer ainsi des conduites à risques.

Le caractère éducatif de l'opération a changé le regard des élèves sur leurs enseignants et sur eux-mêmes, l'inscription dans cette démarche a également démontré que la posture d'un adulte éducateur peut se transformer, et surtout, être « travaillée » :

- en distinguant produit et conduite dans une démarche de lutte contre les drogues et la toxicomanie ;

- en faisant se compléter instruction et éducation dans l'école ;

- en utilisant une pédagogie de l'environnement comme vecteur de bien-être, c'est à dire une éducation relative à l'environnement qui participe d'une certaine forme d'insertion sociale.

\section{BIBLIOGRAPHIE}

Ballion, R. (1999). Séminaire « Éducation à la santé : conduites d'excès, conduites d'essai ». Vidéocassette. Toulouse : ENFA.

Cottereau, D. (1994). À l'école des éléments. Lyon : Chronique sociale. École et Nature. (1998). Une méthode d'éducation à l'environnement, la pédagogie de projet. Paris : L'Harmattan.

MILDT (Premier Ministre). (1999). Plan triennal de lutte contre la drogue et de prévention des dépendances : 1999-2000-2001. Paris : MILDT.

Parquet, P. (1999). Séminaire «Éducation à la santé : conduites d'excès, conduites d'essai ». Vidéocassette. Toulouse : ENFA.

\section{NOTES}

1. Une précédente recherche-action menée de 1997 à 1999 sur l'éducation à la citoyenneté nous avait également permis d'identifier à la fois des problématiques sociales liées à la santé, ainsi que des adultes en demande d'accompagnement.

2. Pour approfondir la question, voir École et Nature (1998). 


\section{AUTEUR}

\section{DAVID KUMURDJIAN}

David Kumurdjian est titulaire d'un BTS protection de la nature et d'une maîtrise de sciences de l'éducation (Université Paul Valéry de Montpellier). Enseignant-formateur au Centre d'Expérimentation Pédagogique de Florac (France) depuis 1991 dans les domaines de l'éducation relative à l'environnement et de la pédagogie appliquée à l'enseignement et à l'animation, il est membre actif du réseau École et Nature depuis 1992 et coorganisateur avec l'Ifrée et le Rectorat de Poitiers du premier colloque français de recherche en ERE (Poitiers, 2000). 